\title{
Striped-Y redux: Redesigning pictographic notational systems for the digital age
}

\author{
Alexander C. Allori* \\ Assistant Professor of Surgery, Duke Cleft \& Craniofacial Center, Division of Plastic, Maxillofacial \& Oral Surgery, Duke Children’s Hospital \& Health Center, \\ Durham, NC, USA
}

\begin{abstract}
Background: Before the advent of electronic medical records, pictographic notational tools were commonly used to succinctly describe clinical phenotypes in paper charts. Over the past 30 years, these methods have been largely abandoned in favor of alphanumerical notational systems, which are more conducive to data entry in clinical and administrative databases. However, such coding systems are incredibly complex, not easily readable or interpretable, and are often limited in their ability to capture phenotypic variation. The purpose of this study was to re-assess the value of pictographic notation for one specific use case - cleft lip/palate (CL/P) - and to propose a novel adaptation of this analog tool for digital data entry.

Methods: An expert focus group was convened and consisted of multidisciplinary clinicians and academicians from several well-known cleft teams. Semi-structured interviews were used to explore familiarity with pictographic notational systems for CL/P, such as Kernahan's "striped-Y" diagram, and to identify how a digital equivalent of these pictograms might best augment modern electronic medical recordkeeping.

Results: Pictographic notational systems found to be used clinically in very few centers, but they retained a role in teaching and facilitating communication. There was considerable variation in accuracy and quality of completion of the pictograms, either due to lack of familiarity with the diagram or rushed completion of the diagram. Experts considered the striped-Y diagram to be a valid, concise, and helpful method for description of CL/P phenotypes. Respondents also voiced frustration regarding existing alphanumeric coding systems (such as ICD, SNOMED, and BPA/CDC). Based on this feedback, a novel digital pictographic system was conceptualized as a graphical user interface to facilitate accurate documentation of clinical phenotypes and selection of proper corresponding alphanumerical codes from various coding schemes.
\end{abstract}

Conclusion: The striped-Y motif translates well for use as a graphical user interface for data entry. With increased availability of touch-capable mobile devices and interactive web apps, this type of custom-designed user interface may improve accuracy and efficiency of data capture.

\section{Introduction}

In the era of paper charting in health care, it was common for clinicians to utilize schematic diagrams to summarize clinical findings. This was widely practiced in the case of birth defects, as anatomic involvement could vary considerably from one patient to the next. One such example is cleft lip and/or palate $(\mathrm{CL} / \mathrm{P})$, in which the lip, nose, dental arch (alveolus), and palate are involved in different combinations and to varying grades of severity. To more accurately record the phenotypic presentation for a child with $\mathrm{CL} / \mathrm{P}$, clinicians would depict the cleft type using a method of pictographic notation [1]. Several methods of pictographic notation have been proposed, but the most popular form was the "striped-Y logo" introduced in 1971 as a graphical representation of the Kernahan \& Stark system of classification $[2,3]$. Several variants were subsequently described (Figure 1).

With the advent of electronic medical records, pictographic notational systems like the striped-Y diagram were all but abandoned. Attempts to translate the striped-Y to the "digital age" have been difficult. This study sought to clarify the role of the striped-Y diagram in contemporary cleft care and to identify whether and how it might be adapted for use with modern electronic health systems.

\section{Methods}

Three complementary approaches were taken in order to identify optimal goals and design parameters for a digital method of pictographic notation for CL/P: (1) evaluation of existing coding schema and other notational systems to identify what level of phenotypic detail needed to be captured; (2) an expert focus group to explore the utility of striped-Y diagrams to cleft teams; and (3) review of two clinical database systems where translation of the striped-Y diagram was attempted with limited success.

Evaluation of classification systems, coding schema, and notational systems for $\mathrm{CL} / \mathrm{P}$

All methods of pictographic notation proposed for CL/P were identified by a MEDLINE query. Critical appraisal of cleft classification systems was previously performed [4]. Additionally, administrative coding schema such as the International Classification of Diseases

Correspondence to: Alexander C. Allori, MD, MPH, Assistant Professor of Surgery, Duke Cleft \& Craniofacial Center, Division of Plastic, Maxillofacial \& Oral Surgery, Duke Children's Hospital \& Health Center, Durham, NC, USA, Tel: 919-668-3110, Fax: 919-684-2670 E-mail: Alexander.allori@ dm.duke.edu

Key words: cleft lip, cleft palate, striped-Y, classification, coding, user interface (UI), user experience (UX), electronic medical record (EMR), electronic health record $(E H R)$

Received: May 18, 2017; Accepted: May 30, 2017; Published: June 02, 2017 


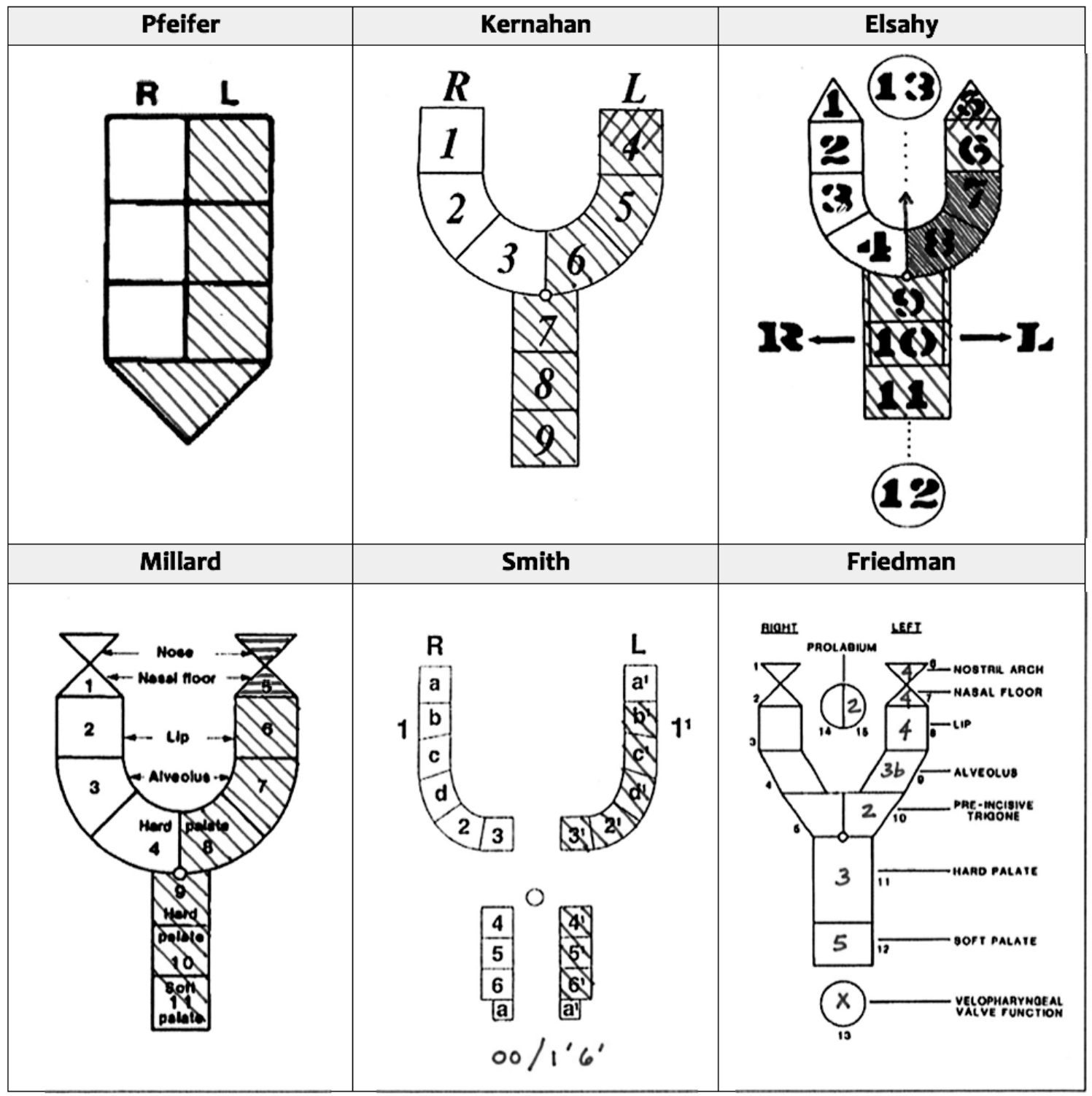

Figure 1. Two original pictographic tools for diagramming cleft phenotypes are by Pfeifer (1966) and Kernahan (1971, revised 1973). Elsahy (1973), Millard (1977), and Smith et al. (1998) described modifications. Friedman et al. (1991) utilized the Y motif in developing an "infographical" tool for semi-quantitatively conveying severity. All diagrams in this figure depict the same phenotype: i.e., left unilateral complete cleft lip and alveolus with a cutaneous (Simonart) band and Veau-III cleft palate. (Pictograms adapted from original sources.)

$9^{\text {th }}$ and $10^{\text {th }}$ clinical modifications (ICD-9-CM and ICD-10-CM), ontologic frameworks such as Systematized Nomenclature of Medicine-Clinical Terms (SNOMED CT), and specialized coding extensions such as the British Pædiatric Association/Centers for Disease Control and Prevention (BPA/CDC) and the ICD-10Based Expanded Code Set for Cleft Research and Surveillance were also studied [5-7]. These sources were used to identify the level of phenotypic detail that could be expressed via commonly used coding systems, identify the aspects of phenotypic description that were typically lost in encoding, and thereby conceptualize the ideal role that a pictographic notational system would play as an adjunct to existing text-based encoding.

\section{Expert Focus Group}

Semi-structured interviews were conducted with 21 members from 13 well-known North American cleft teams. Disciplines represented included plastic surgery, oral/maxillofacial surgery, otorhinolaryngology, dentistry, speech/language pathology, audiology, nursing, prenatal ultrasonography, and team coordination/ administration. Participants were asked to expound upon their degree of familiarity with pictographical notational systems and the role these tools presently play in the daily workflow of their teams. Sample clinical documentation from a subset of centers was reviewed to identify methods of documentation and the manner of diagramming with any pictograms. Participants were also observed in the process of manually diagramming using various pictographic notational systems; they were asked to verbalize their thoughts on the strengths and weaknesses of each system. Data were interpreted qualitatively to map recurrent themes to desirable/undesirable characteristics of a pictographic notational system. Potential obstacles to implementation of pictograms in contemporary digital workflows, and strategies for overcoming those obstacles, were also explored. 


\section{Review of prior attempts to adapt the striped-Y diagram for data entry}

Through discussions with members of the American Cleft PalateCraniofacial Association (ACPA), we identified electronic databases used by some teams where the striped-Y motif was adapted as a means of data entry. The strengths and weaknesses of these implementations were appraised, especially with regard to usability and in light of the desirable/undesirable characteristics identified by the focus group, above.

\section{Results}

\section{Summary of Focus-Group Findings}

Recurrent themes expressed by participants are summarized below:

1. Photographs are an indispensable component of the contemporary medical record and remain the "gold standard" for fully and accurately documenting the cleft phenotype.

2. Pictograms are a useful and concise method of depicting the cleft phenotype.

3. Pictograms are superior to many text-based classification or coding systems for accuracy and completeness of phenotypic description; however, pictograms are inferior to encoding for informatical purposes (e.g., for querying, grouping, and analyzing data).

A critique of the diagramming process also revealed the following:

4. There is great variability in style of usage of the pictograms; that is, they are prone to idiosyncratic personalization and misuse such that different clinicians might depict the same cleft in slightly different ways.

5. Pictograms are prone to under-description due to haste in completion.

6. Pictograms are also prone to misunderstanding. Accurate interpretation of pictograms depends on user experience with the particular system employed.

\section{Photography is the "gold standard" for documentation}

Experts unanimously agreed that photographs are an extremely valuable form of clinical documentation. There was great variability in the method of photographic acquisition. ${ }^{\dagger}$ The role of photography was notably distinct from that of the pictogram: whereas the photograph was primarily a method of documentation and comprised part of the medical history, the pictogram corresponded more to an exam-based assessment of the patient. In addition, multiple photographs were often needed to fully capture the phenotypic details - i.e., intraoral

†Teams possessing a "media room" attached to their clinical space reported that they were able to routinely obtain and catalog photographs in all cases. Teams that rely on a hospital-wide "media department" reported that photographs were usually (but not always) obtained when ordered. Interestingly, most teams lacked specific protocols to guide the systematic acquisition of clinical photographs for CL/P. Hospital-acquired photographs were reported to be variable with regard to proper positioning, whereas team-run mediaroom photographs were usually taken according to a stricter protocol. In both settings, photographs always included the frontal views, usually profile views, sometimes submenta views, but infrequently three-quarter views. Three-dimensional photographs, if available, were considered ancillary and did not supplant two-dimensional photographs. Photographs were taken during facial repose and only rarely during smiling. Intraoral dental details were photographed in older children, but the palate was not usually photographed in infants. Respondents noted that photographs were often stored separately from the rest of the medical record, which created friction in the workflow if a clinician wanted to review prior photographs. Some respondents also identified this as a barrier to access: e.g., "I know photographs are taken, but I have no clue where they're stored or how to access them." photographs are required to document the state of the palate, dental arch (alveolus), and teeth, and facial photographs are required to document the state of the lip and nose. Thus, while photography was considered the "gold standard" for documentation, it did not seem to fully replace pictograms.

\section{The striped-Y motif is familiar to clinicians - but specific diagrams can be misinterpreted}

Clinicians from all disciplines were generally familiar with the concept of pictographic notation to describe cleft phenotypes. They considered pictographic notation to be one level of abstraction above a photograph - that is, a symbolic representation of essential information necessary for classification and development of a treatment plan.

Generally, surgeons and orthodontists expressed the most comfort with pictographic notational systems, compared with their colleagues from other disciplines. Several non-surgeon members of cleft teams admitted to having "no idea" how to interpret a striped-Y diagram. The ability of a respondent to properly interpret sample pictograms correlated with self-report of prior exposure to and experience with the specific system being demonstrated. (Type and extent of experience were not explored in detail.) Of the specific pictographic notations that were demonstrated (Figure 1), the traditional Kernahan striped-Y was the most recognizable, followed by the Millard modification. Respondents expressed some concern that, unlike a photograph, a pictogram might not be universally or completely understood.

\section{Pictograms can accurately depict essential morphologic details - but are prone to misuse}

In our survey of 13 cleft teams, only two teams still actively utilized pictographic notation in their clinical charting. One team employed the original Kernahan striped- $Y$, and the other a "proprietary" modification of the Millard striped-Y diagram. Review of sample charts from participating centers revealed that several methods of phenotypic notation were being used (Figure 2). These methods were able to accurately record detailed anatomic information in many cases; sometimes, handwritten or typed annotations were made to clarify specific phenotypic peculiarities that the user wanted highlighted (e.g., cutaneous band, notched alveolus, relatively large alveolar gap, arch collapse, etc.). In the two centers that still employed pictograms, clinicians admitted to "sometimes rushing" to fill-in the diagram. This was especially true if there was redundancy in documentation (i.e., the team would use several alternate methods of phenotypic description, such as checkboxes, text-based classifications, or codes that made the "extra" pictogram seem superfluous and unnecessary) (Figure 2). In some cases, the pictogram was ignored and left blank.

A subset of experts was also asked to use Kernahan and Millard diagrams to pictographically encode sample cases. There was considerable variation in accuracy, completeness, and quality of these depictions. Most users strayed from "official protocol" described in the original publications and introduced idiosyncratic modifications. Some anatomic details were under-rated (e.g., cutaneous band represented as an incomplete cleft) and others were over-rated (e.g., notched alveolus as a complete defect). Upon further exploration, experts explained that they would "normally write notes in the margin" to clarify such details or to summarize the phenotype (e.g., "LUCCL/P"; cf. Figure 2-D). Users did well reinterpreting their own completed striped-Y diagrams, but interpretation of diagrams completed by others was often inaccurate.

The most common point of confusion expressed by respondents related to the denotation of different methods of shading (stippling, 
(A)
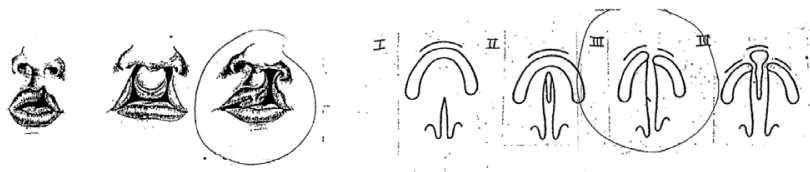

(B)

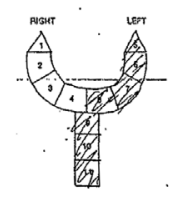

(C)

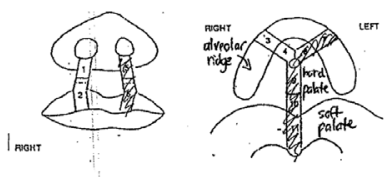

(D)

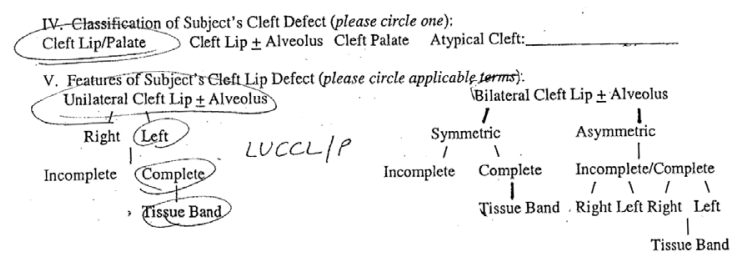

(E)

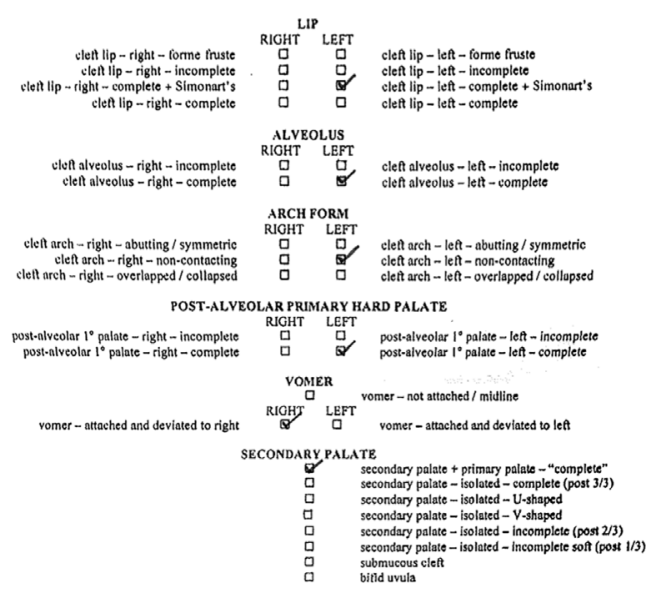

(F)

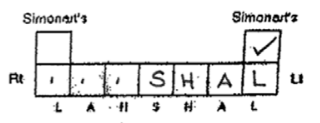

Figure 2. Several methods of cleft documentation, sampled from data forms used by various institutions: (A) set of illustrations depicting common forms of CL/P; the appropriate form for a particular patient was circled. (B) variation of the striped-Y pictogram, which was shaded to denote the cleft morphology. (C) schematic representation of normal anatomy upon which the cleft morphology was shaded. (D) taxonomical hierarchy; the appropriate terms were circled, and a summary abbreviation "LUCCL/P" (left unilateral complete cleft lip and palate) was hand-written. (E) checkboxes for characterizing each component of the phenotype. (F) modified LAHSHAL notation.

diagonal lines, horizontal lines, cross-hatching, blackening). The second-most common uncertainty regarded "which box in the diagram corresponds to which anatomic structure?" With the Millard modification, specifically, participants voiced uncertainty with how to properly fill-in the unnumbered, upside-down triangles at the top of each fork of the Y. (Per protocol, parallel lines should be used to represent the nasal deformity, and the density of those parallel lines should reflect the severity of the deformity [8].) Respondents were divided on the utility of the Elsahy modification, viewing the construction of the $\mathrm{Y}$ itself quite favorably but expressing uncertainty as to how to properly utilize the circles depicting the premaxilla (\#13), the arrows labeled $R$ and $L$, and the circle representing the pharynx (\#12). (Per protocol, an arrow should be drawn to represent premaxillary protrusion. The rightward or leftward arrows should be circled to represent palatal segmental collapse. The pharyngeal circle is intended to be updated at an older age to describe the pattern of pharyngeal closure [9].) None of the respondents in this study was familiar with the Smith et al. or Friedman et al. modifications [10,11]. After explanation of these methods, respondents generally thought the Smith pictographic diagram too confusing and thought the Friedman infographic diagram both too cumbersome for recording a new diagnosis and too difficult to interpret a previously recorded diagnosis.

\section{Literal translation of the striped-Y diagram for data entry has been met with mixed success}

In their EMR systems or proprietary databases, most cleft teams have abandoned pictographic notation entirely in favor of more "computer-friendly" forms comprised of checkboxes and text fields. However, two cleft-specific databases were identified as having attempted to adapt the striped-Y diagram as a means of data entry: the proposed database by the ACPA Data Standards Committee - a project that was ultimately abandoned - and the Crux Cleft Database used by a cleft team in Australia (Figure 3). From these examples, it is evident that the premise of a digital adaptation of the striped-Y diagram feels familiar, as it recalls the original pictographic notational systems; however, the specific methods of translation to digital forms were problematic in both cases. Instead of depicting cleft features visually by shading the regions of the diagram, each anatomic component requires clicking a checkbox or button to designate presence and severity of a defect. Thus, in these implementations, the ease-of-use of the original simple pictograms is lost, and inefficiency is introduced. Interpreting the patterns of discrete clicked checkboxes or buttons is much less clear than seeing continuous shading on a diagram. To the teams' credit, these limitations are likely reflective of technological constraints at the time that the data-entry forms were developed.

Interestingly, the Crux Cleft Database was quite innovative in overlaying the Y diagram on top of a schematic representation of the lip, dental arch (alveolus), and palate. In this manner, it attempted to reduce confusion regarding which part of the diagram corresponded with which part of anatomy, and thus improve inter-rater reliability for usage and interpretation.

\section{Alphanumeric-based phenotypic encoding is more appropriate for informatics}

It is interesting that while some experts waxed nostalgic for an era when the striped- $Y$ appeared on the inside cover of every chart, each nevertheless has adapted to the new digital workflow, and none has expressed a dire need to reintroduce the striped-Y per se into clinical practice. Most respondents believed the combination of clinical photography and text-based description to be superior to pictographic notation. This highlight one weakness of pictographic notation: While pictograms work well for summarizing intricate anatomic details of an individual case, they are not conducive to sorting cases into classes, categories, or cohorts - as is often required for research, administration, 


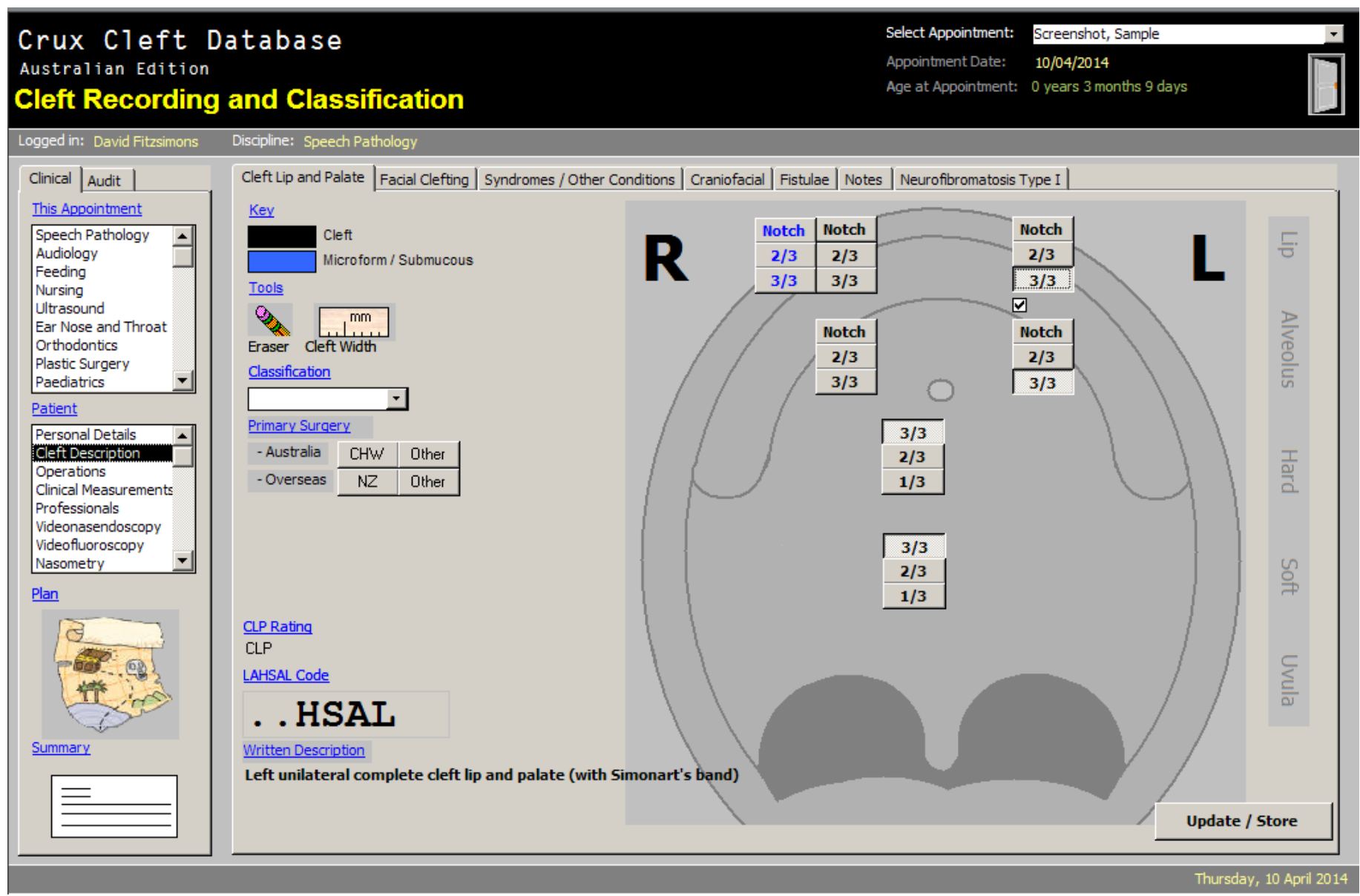

Figure 3. Data-entry display from the Crux Cleft Database v. 10 (C2010, David Fitzsimons). The selector buttons corresponding to lip, alveolus, primary (pre-foraminal) palate, and secondary (post-foraminal) hard and soft palate are laid out in a Y-shaped configuration, recapitulating the classic striped-Y diagram. Innovatively, the Y pattern was superimposed on a schematic of normal anatomy to improve orientation and comprehension.

and quality improvement. Succinct text-based codes are likely more useful to this purpose. Be that as it may, most respondents still thought pictograms to have some kind of intrinsic value. Perhaps certain conditions, such as $\mathrm{CL} / \mathrm{P}$, are best described visually - it is arguably easier to interpret, visually, a striped-Y diagram than it is to decipher the corresponding ICD diagnostic codes or CLAP or LAHSHAL notational descriptions.

\section{Discussion}

\section{Adapting the striped-Y for the digital age}

With traditional analog tools, such as hardcopy diagrams, there is arguably some tension between the desire for comprehensiveness and the need for simplicity. Consider, for example, the various methods shown in Figure 1. There is an inherent beauty in the minimalistic simplicity of Pfeifer's original pictogram. As the desire arose to include more and more detail pictographically, many variations were introduced, and these evolved to the point of becoming cumbersome note-taking devices. Kernahan was quite vocal in his criticism of including too many clinical details, lest the result be an overly-complex diagram that is "incapable of revealing at a glance just what the original deformity consisted of [sic]" [11]. Translation of these motifs to the digital world opens up new possibilities where the user interface remains visually simple and intuitive, but other hidden layers may still capture extreme levels of detail.
We propose a new role for the striped-Y in the digital age: as a graphical user interface. As such, the diagram can serve both as an intuitive method of input (guided data entry) and as a familiar method of output (depiction of cleft type for later reference). As such, it retains the strengths and simplicity of the analog striped-Y diagram while also harnessing the potential of a digital interface, such as interactivity and alternate forms of data visualization.

Figure 4 demonstrates the conceptual design for a novel "anatomic-Y" for use as a graphical user interface. Based upon the striped-Y paradigm, it consists of two limbs representing the nasal floor, cutaneous lip, red lip, alveolar process, and primary palate; and a central section representing the secondary hard palate, and soft palate. In traditional striped-Y variants, the cells (stripes) represent parts of the anatomy and are numbered for reference. Respondents in the focus group interviews found the various numbering schemes used across the striped-Y variants to be inconsistent and confusing. The "anatomic- $Y$ " abandons cell numbering/labeling and instead superimposes the wireframe pattern upon an illustration of normal anatomy for reference. As cells are shaded, the anatomic part that is clefted is immediately apparent. Moreover, the curvilinear shape of the wireframe is designed such that shading of the relevant cells will simulate the shape of the anatomic defect. For example, representation of a Veau-II cleft palate shows a central defect with midline, exposed vomer, whereas a Veau-III cleft palate shows a unilateral defect with 

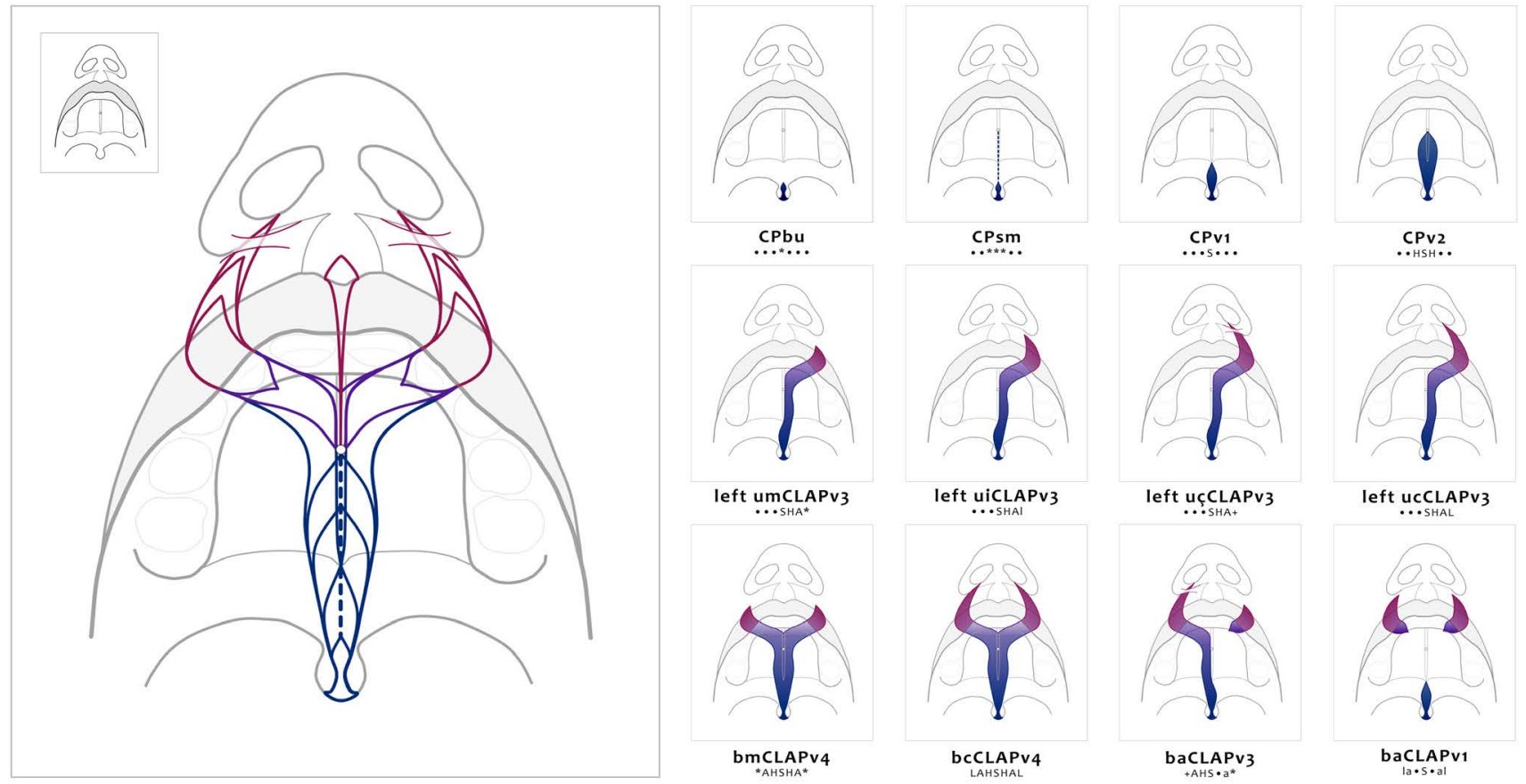

Figure 4. The "anatomic-Y" diagram superimposes a Y-shaped wireframe on a gray schematic that realistically depicts normal nasolabial and intraoral features with a somewhat threedimensional perspective. The curvilinear form of the Y wireframe is specifically designed such that shading appropriate cells creates the morphologic form of the cleft phenotype. When used as a graphical user interface, the complex wireframe is hidden from the end-user so that only the shaded pattern is seen. Example phenotypes from left-right and top-down, with corresponding CLAP and LAHSHAL notations: bifid uvula [CPbu], submucous cleft palate [CPsm], Veau-I cleft palate [CPv1], Veau-II cleft palate [CPv2], left unilateral microform cleft lip and alveolus with Veau-III cleft palate [umCLAPv3], left unilateral incomplete cleft lip and alveolus with Veau-III cleft palate [uiCLAPv3], left unilateral complete cleft lip and alveolus with skin band and Veau-III cleft palate [uçCLAPv3], etc.

attachment of the vomer to the contralateral palatal shelf. Also, a midline labial cell allows for specification of a median cleft lip, a dotted line allows for specification of submucous cleft palate, and bands allow for designation of cutaneous (Simonart) bands. With this level of visual detail, the digital pictogram may be considered to be more illustration than schematic.

In traditional striped-Y variants, severity is specified by differential methods of shading. Respondents in the focus group expressed uncertainty about the significance of various methods of shading (stippling, diagonal lines, horizontal lines, cross-hatching, and blackening) that could be employed. Therefore, the digital pictogram eliminates differential shading; a cell is either "on" (clefted) or "off" (unclefted). To represent severity, each anatomic region is subdivided into different cells. For example, cleft lip can be depicted as complete (by shading all three cells in the lip region), incomplete (lower two cells), or lesser-form (lowest cell). A similar degree of control is possible for cleft palate and cleft alveolus.

Figure 4 demonstrates some of the many types of clefts that can be depicted with this system. The wireframe itself may appear overly complex and foreboding - If it were a traditional analog tool (printed on paper), this would certainly be the case; however, in its implementation digitally, the underlying wireframe is hidden from the user, and only the shaded figure is seen.

Most importantly, this graphical user interface can be exploited to automatically compute the corresponding written descriptions or codes (e.g., LAHSHAL notation, CLAP notation, and ICD-9-CM, BPA/ CDC, ICD-10-CM codes, etc.). This allows the clinician to interact with the computer in a way that makes most sense (visually and tactilely) but to also leverage the power of (auto-generated) text-based notation and codes for administration, billing, data aggregation, and outcomes analysis. Thus, condition-specific user interfaces have great potential to revolutionize data collection - improving efficiency and accuracy while also streamlining the clinical workflow in the process.

Figure 5 depicts a conceptual implementation on a touchcapable, mobile device. (Web-based applications would also be suitable implementations.) The wireframe is hidden from view, and the user is presented only with a diagram of normal anatomy, which should simplify the learning curve and improve the overall user experience. Slider controls allow the user to specify the location and severity of the cleft. As the sliders are moved, corresponding parts of the wireframe become shaded, and the user sees the morphology updated in realtime. In this conceptual design, the programmed logic automatically adjusts the state of the dental arch (alveolus) based on epidemiological likelihood of the phenotype - For example, setting one labial slider to "complete cleft lip" and the palatal slider to "complete cleft palate" would automatically set the alveolar slider to "complete," thus establishing the common phenotype "unilateral complete cleft lip, alveolus, and Veau-III cleft palate" ([ucCLAPv3] or [LAHS•.•] in CLAP and LAHSHAL notation, respectively). Nevertheless, the user has the option to override this automatic setting and manually specify the state of the alveolus, thus allowing depiction of non-contiguous clefts. In the above example (keeping the labial and palatal settings the same), the alveolar slider could be adjusted to "normal" (intact alveolus), resulting in "unilateral complete cleft lip and Veau-II cleft palate" ([ucCLPv2] or [L•HS•.•]). Furthermore, the application would automatically generate the correct phenotypic description, notation (e.g., LAHSHAL and/or CLAP notation [4]), and codes from various codesets (e.g., 


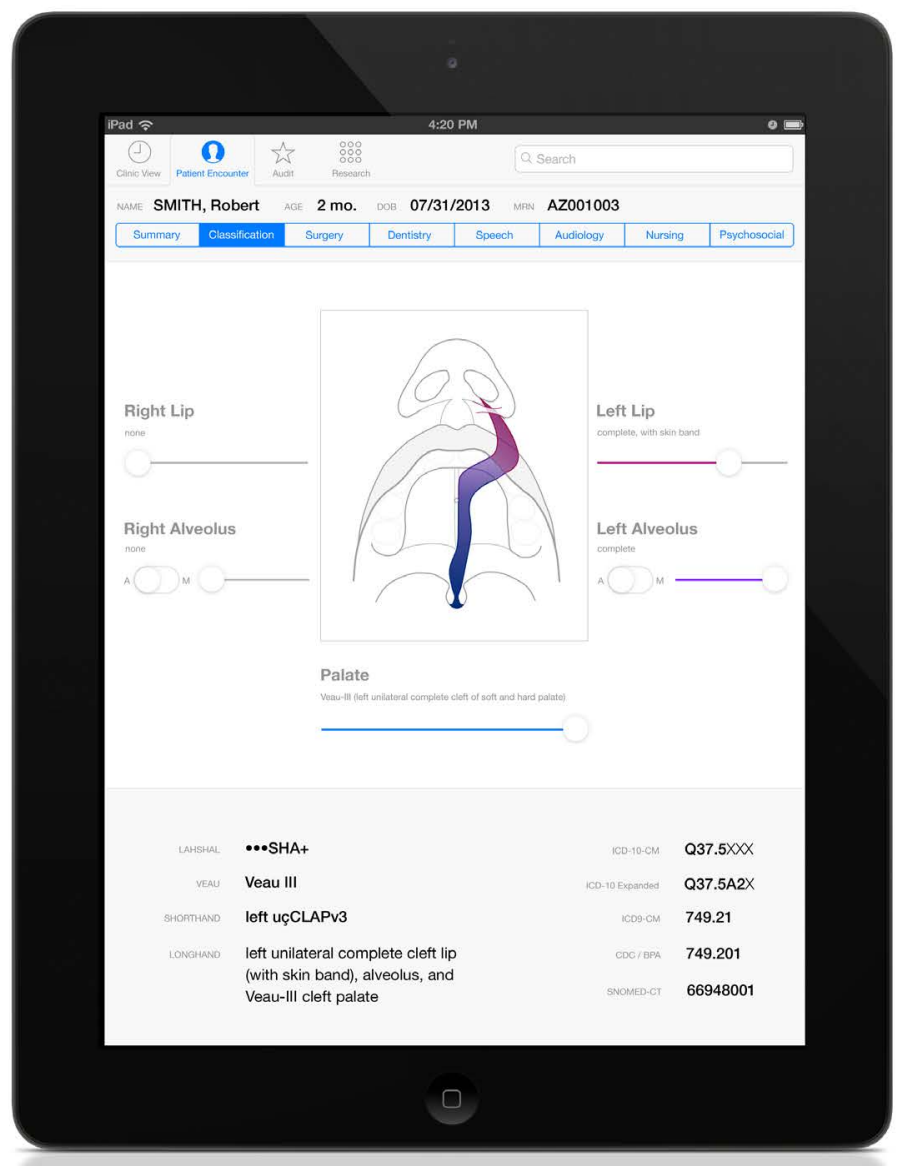

Figure 5. Conceptual design of a touch-based user interface designed for the Apple iPad and intended to streamline digital data entry. The wireframe from Figure 4 is hidden from the user, who only sees normal anatomy as the starting point. To classify the cleft phenotype, the user slides "severity sliders" corresponding to the lip, alveolus, and palate. The center illustration automatically updates, in realtime, to match the phenotype specified by slider controls. In the case depicted, the phenotype specified by the slider controls is a left unilateral complete cleft lip and alveolus with cutaneous band and Veau-III cleft palate.

ICD-10-CM, ICD-10-based expanded codeset, [7] ICD-9-CM, BPA/ CDC, and SNOMED-CT) based on the state of the wireframe in the graphical user interface (Figure 6). This would eliminate the need to manually choose these codes. A webapp version of this interface system might also be integrated into the EMR to automatically populate the problem list in the patient's record with the new ICD-10-CM and/or SNOMED-CT code.

\section{Conclusion}

In the focus group, one team coordinator stated, "We used to have them [striped-Y diagrams] printed on the face sheet in the front of every chart, and it was so convenient. But now everything's on the computer, so we don't use [pictograms] anymore." In this project, we propose a method for translating this convenient analog tool to the digital arena. This system has the potential to improve ease-of-use, accuracy, and reliability with regard to phenotypic classification and coding of $\mathrm{CL} / \mathrm{P}$ in modern electronic medical records.

\section{Acknowledgements}

Many thanks to the following people for their invaluable feedback: David A. Fitzsimons, BS, CCC-SLP; Jeffrey R. Marcus, MD; John G. Meara, MD, DMD, MBA; John B. Mulliken, MD; Patricia Chibbaro, MSN, C-PNP; Bonnie L. Padwa, DMD, MD, Dottie MacDonald, RN; Ann Mabie, CCC-A; and Elizabeth Marquez-Allori, MSN, C-PNP.

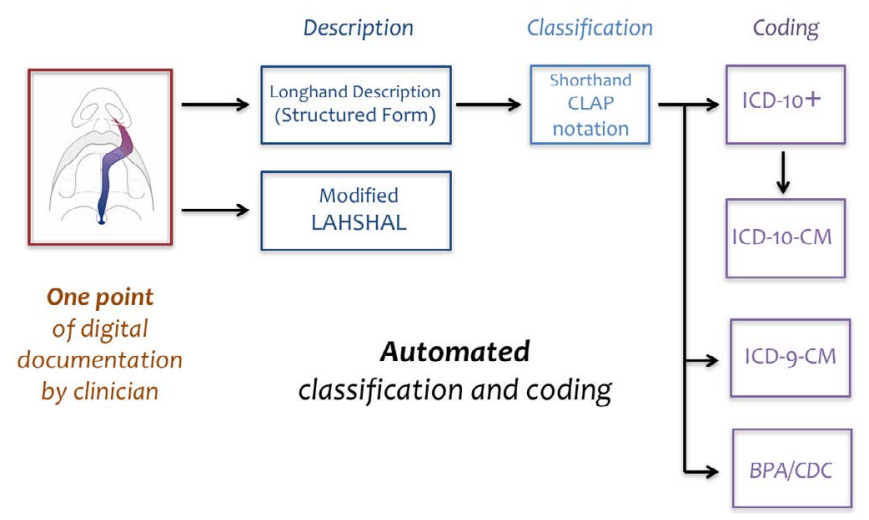

Figure 6. The graphical user interface serves as a digital point of entry for description of the cleft phenotype. Look-up tables then permit the automatic generation of corresponding longhand phenotypic descriptions, shorthand notation (e.g., LAHSHAL notation, CLAP notation), and diagnostic codes (e.g., ICD-9-CM, BPA/CDC, ICD-10-CM, and SNOMEDCT codes), which can be stored in an electronic medical record system, administrative database, or registry.

\section{Disclosures}

The author has no associations, financial or otherwise, that might pose a conflict of interest related to the information presented in this manuscript. 


\section{References}

1. Davis JS, Ritchie HP (1922) Classification of congenital clefts of the lip and palate with a suggestion for recording these cases. JAMA 79: 1323-1327.

2. Kernahan DA, Stark RB (1958) A new classification for cleft lip and cleft palate. Plast Reconstr Surg Transplant Bull 22: 435-441. [Crossref]

3. Kernahan DA (1971) The striped Y--a symbolic classification for cleft lip and palate Plast Reconstr Surg 47: 469-470. [Crossref]

4. Allori AC, Mulliken JB, Meara JG, Shusterman S, Marcus JR (2017) Classification of cleft lip/palate: Then and now. Cleft Palate Craniofac J 54: 175-188. [Crossref]

5. Aylsworth AS, Allori AC, Pimenta LA, Marcus JR, Harmsen KG, et al. (2015) Issues involved in the phenotypic classification of orofacial clefts ascertained through a state birth defects registry for the north carolina cleft outcomes study. Birth Defects Res A Clin Mol Teratol 103: 899-903. [Crossref]
6. Allori AC, Cragan JD, Della Porta GC, Mulliken JB, Meara JG, et al. (2015) Clinician's primer to ICD-10-CM coding for cleft lip/palate care. Cleft Palate Craniofac J 54: e7-e13. [Crossref]

7. Allori AC, Cragan JD, Cassell CH, Marcus JR (2016) ICD-10-based expanded code set for use in cleft lip/palate research and surveillance. Birth Defects Res A Clin Mol Teratol 106: 905-914. [Crossref]

8. Millard RD Jr. (1976) The naming and classifying of clefts. In: Cleft Craft. Boston, 41-55.

9. Elsahy NI (1973) The modified striped Y--a systematic classification for cleft lip and palate. Cleft Palate J 10: 247-250. [Crossref]

10. Smith AW, Khoo AK, Jackson IT (1998) A modification of the Kernahan "Y" classification in cleft lip and palate deformities. Plast Reconstr Surg 102: 1842-1847. [Crossref]

11. Friedman HI, Sayetta RB, Coston GN, Hussey JR (1991) Symbolic representation of cleft lip and palate. Cleft Palate Craniofac J 28: 252-259. [Crossref]

Copyright: $\odot 2017$ Allori AC. This is an open-access article distributed under the terms of the Creative Commons Attribution License, which permits unrestricted use, distribution, and reproduction in any medium, provided the original author and source are credited. 